\title{
Commentary
}

\section{Balancing the Promise and Risks of Ketamine Treatment for Mood Disorders}

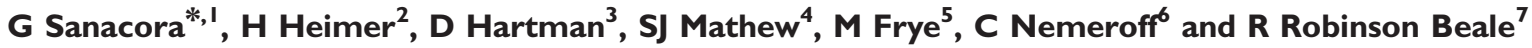 \\ 'Yale Depression Research Program, Yale University School of Medicine, New Haven, CT, USA; '2Cure Alliance for Mental Illness, Providence, RI, \\ USA; ${ }^{3}$ Ketamine Advocacy Network, Seattle, WA, USA; ${ }^{4}$ Baylor College of Medicine, Houston, TX and Michael E. Debakey VA Medical Center, \\ Houston, TX, USA; ${ }^{5}$ Mayo Clinic, Rochester, MN, USA; ' University of Miami, Miami FL, USA; ${ }^{7}$ Blue Cross of Idaho, Boise, ID, USA
}

Neuropsychopharmacology (2017) 42, II79-II8I; doi:I0.1038/npp.2016.193; published online 12 October 2016

A growing number of small but highly consistent controlled trials, case series, and anecdotal reports have demonstrated ketamine's rapid and robust transient antidepressant properties. A meta-analysis of the published studies recently completed by the American Psychiatric Association Council of Research Task Force on Novel Biomarkers and Treatments found the data from these studies to provide 'compelling evidence that the antidepressant effects of ketamine infusion are both rapid and robust, albeit transient.' (Newport et al, 2015). Although there are few reports examining the efficacy of repeated ketamine administration, those that have been published suggest the initial antidepressant effect can be extended over several weeks with repeated administrations (Murrough et al, 2013; Singh et al, 2016). Accounts of these findings have generated great interest in the media and optimism among patients. This has led to a growing number of clinics and practitioners who are now offering various forms of ketamine treatment for mood and anxiety disorders throughout the United States, and a growing advocacy network that promotes the accessibility of the treatment (ketamineadvocacynetwork.org).

However, there is concern by some that the enthusiasm engendered by these reports combined with the desperate plight of the patients and their families may be leading to use of ketamine in ways that are not yet adequately supported by the existing evidence. Although there is strong evidence that treatment with ketamine can provide significant short-term benefits to a large proportion of individuals suffering from serious, disabling and potentially fatal mood disorders, the treatment has not yet undergone the test of larger-scale clinical trials to demonstrate the durability and safety of long-term treatment. Neither has the treatment approach been subjected to the scrutiny of a US FDA review. In addition, the growing abuse of ketamine in Asia and other areas of the world (Advisory Council on the Misuse of

\footnotetext{
* Correspondence: Dr G Sanacora, Yale Depression Research Program, Yale University School of Medicine, 100 York St Suite 2], New Haven, CT 065II, USA, Tel: +203 974 7535, Fax: +203764 5963, E-mail: gerard.sanacora@yale.edu

Accepted article preview online 19 September 2016
}

Drugs) has raised significant concerns over the potential for fostering substance use disorders and drug diversion through the broader use of ketamine in the treatment of depression. Consequently, the medical field is now somewhat at odds over the expanding off-label use of ketamine in the treatment of mood disorders.

A group of academic researchers, community clinicians, policy makers, and patient advocates recently convened to discuss this topic. There was general agreement that ketamine may be unique among available medications in its ability to rapidly help patients suffering with severe treatment-resistant depression, and given the known risks associated with prolonged severe major depressive episodes, efforts should be made to further study the risks and benefits of the treatment, and to support the responsible use of ketamine for treating carefully selected patients in appropriate settings. In order to best balance the promise of this treatment and the potential risks associated with its widespread use in the clinical setting, we recommend that the following three steps should be taken without delay.

1. Creation of a Joint Statement from professional organizations with expertise in related areas of study, providing a clear and concise overview of the state of the field. This statement should provide clinicians and patients with clear information based on the existing evidence on the efficacy and risks of ketamine treatment of mood disorders. This information could be used to educate clinicians and assist in the informed consent process of prospective patients. The statement should also formulate some general (minimal) standards of practice, based on existing data and the consensus of experts in the field, to afford some guidance to clinicians interested in providing the treatment option and assisting patients with referrals. This should include evaluation and screening criteria, safety considerations, and alternatives to such care.

2. Formation of a Registry of Patients receiving ketamine for the treatment of mood disorders. As racemic ketamine no longer has clear patent protections, it is unlikely that any large-scale phase II or III clinical trials will be completed for an FDA review. The formation of a registry appears the next 
best way of capturing data on the real-world effectiveness and safety of the treatment as it is being provided through various clinics around the country. This registry would serve to provide new data for ongoing analyses to help better understand the effects of dose, route of administration, frequency of dosing, duration of response, rates of adverse events, and to track any potential serious adverse events. Moreover, the registry could also provide a measure of the number of patients being treated with this approach and will allow clinicians to track patients' past or current enrollment in other ketamine treatment programs.

3. Support of Continued Research. As outlined above, several important questions regarding the longer-term efficacy and safety, the optimal dose and route of administration, and the mechanism of antidepressant action of ketamine for the treatment of mood disorders remain incompletely answered. These areas remain topics of intense investigation at many academic sites. A review of clinicaltrials.gov listings on 26 April 2016 shows 28 studies currently enrolling subjects using the search terms ketamine and major depressive disorder, and there are several additional studies investigating the effects in related diagnoses, such as bipolar disorder, social phobia, post-traumatic stress disorder and obsessive-compulsive disorder, and alcohol and substance use disorders. The NIMH is currently funding both intramural and extramural research on this topic including a ketamine dose finding study (clinicaltrials.gov NCT01920555); a Department of Veterans Affairs study similar in scope is also underway. However, these studies are unlikely to yield the large sample sizes that are required to provide definitive results regarding clinically important issues such as moderators of response, or to provide high levels of confidence on safety issues, especially related to the longer-term use of the drug or risks for abuse. Some of this large sample size work is currently underway with related compounds such as intranasal esketamine $(\mathrm{S}(+)$ enantiomer of ketamine) that has received a 'breakthrough therapy designation' from the US FDA. These studies will provide critical information on the longer-term safety and efficacy of that specific compound. However, the generalizability of these findings to other forms and routes of ketamine administration remains in question. Therefore, additional mechanisms of research support, either through other governmental agencies, philanthropic foundations, or health care organizations, are needed to fund research projects assessing these questions.

Other forms of research specifically designed to elucidate ketamine's mechanism of antidepressant action could prove extremely valuable in helping not only guide the development of novel pharmaceutical interventions but also advancing the basic understanding of the pathophysiology of mood disorders. At present the majority of studies have focused on ketamine's action on the N-methyl-D-aspartate (NMDA) receptor, where it functions as a non-competitive antagonist. A growing number of preclinical studies provide strong evidence that this action and an ensuing cascade of effects, involving increased neurotrophic factor activity, and changes in synaptic plasticity are critical in generating an antidepressant-like response in rodent models (Duman et al, 2016). However, while some clinical studies examining the antidepressant efficacy of other drugs that act as NMDA receptor antagonists have reported positive findings, others have not (Newport et al, 2015). The inconsistencies in the clinical findings have led some to question the extent to which NMDA receptor antagonism actually mediates ketamine's mechanism of rapid antidepressant action (Sanacora and Schatzberg, 2015). Ketamine is known to have a broad range of effects on the central nervous system (ie, modulation of opioid, dopaminergic, and serotonergic systems) as well as having effects on inflammatory processes, and findings from a recent study suggests that a metabolite of ketamine, that has minimal or no affinity for the NMDA receptor, may in fact be responsible for a majority of the antidepressant-like responses in a mouse model (Zanos et al, 2016). At this point, it would seem wise to also study mechanisms of ketamine action other than NMDA receptor modulation. A greater understanding of the underlying mechanism of action could lead to the development of novel therapeutics with markedly improved risk/benefit ratios.

In conclusion, balancing the hope that ketamine brings to patients suffering from the debilitating effects of severe mood disorders, with the limited data on the longer-term effectiveness and potential risks associated with the treatment approach, is a growing challenge faced by clinicians treating these patients. Considering the likely fact that there will not be the usual large-scale phase 3 pivotal trials performed with racemic ketamine in order to receive US FDA indication, we recommend specific steps be initiated to increase the collection of data on the effectiveness and safety of the treatment, and to provide fair, balanced, and accurate information to patient and clinicians considering the treatment option.

\section{FUNDING AND DISCLOSURE}

GS has received consulting fees from Allergan, Alkermes, AstraZeneca, Avanier Pharmaceuticals, BioHaven Pharmaceuticals, Bristol-Myers Squibb, Hoffman La-Roche, Janssen, Merck, Naurex, Novartis, Noven Pharmaceuticals, Servier Pharmaceuticals, Taisho Pharmaceuticals, Teva, Valeant, and Vistagen therapeutics over the last 36 months. He has also received additional research contracts from AstraZeneca, Bristol-Myers Squibb, Eli Lilly, Johnson \& Johnson, Hoffman La-Roche, Merck, Naurex, and Servier over the last 36 months. Free medication was provided to GS for an NIH-sponsored study by Sanofi-Aventis. In addition, he holds shares in BioHaven Pharmaceuticals Holding Company and is a co-inventor on a patent 'Glutamate agents in the treatment of mental disorders'. Patent number: 8778979. SJM has received consulting fees from Acadia, Alkermes, Cerecor, Naurex, Otsuka, Teva, Valeant, and Vistagen Therapeutics. He has received research contracts from Janssen Research \& Development and Otsuka. He was also supported by the Johnson Family Chair for Research in Psychiatry at Baylor College of Medicine and through the use of resources and facilities at the Michael E. Debakey VA Medical Center, Houston, TX. MF has received consulting fees from Janssen Research \& Development, Mitsubishi Tanabe Pharma Corporation, Myriad Genetics, Neuralstem, Sunovion, Supernus Pharmaceuticals, Teva Pharmaceuticals, and CME/Travel Support from the American Physician Institute and CME Outfitters. $\mathrm{CN}$ has received consulting fees from Xhale, Takeda, Mitsubishi Tanabe Pharma Development America, Taisho Pharmaceutical, Lundbeck, 
Prismic Pharmaceuticals, Bracket (Clintara), Total Pain Solutions (TPS), Gerson Lehrman Group (GLG) Healthcare \& Biomedical Council, Fortress Biotech, Sunovion Pharmaceuticals, Sumitomo Dainippon Pharma, and Janssen Research \& Development. He is a stockholder in Xhale, Celgene, Seattle Genetics, Abbvie, OPKO Health, Bracket Intermediate Holding, and Network Life Sciences over the last 36 months. He has served on the scientific advisory boards of American Foundation for Suicide Prevention (AFSP), Brain and Behavior Research Foundation (BBRF) (formerly named National Alliance for Research on Schizophrenia and Depression (NARSAD)), Xhale, Anxiety Disorders Association of America (ADAA), Skyland Trail, Bracket (Clintara), RiverMend Health LLC, and the Laureate Institute for Brain Research. He is on the board of directors of AFSP, Gratitude America, and the $\mathrm{ADAA}$. $\mathrm{CN}$ has received income sources or equity of $\$ 10000$ or more from the American Psychiatric Publishing, Xhale, Bracket (Clintara), CME Outfitters, and Takeda. He also holds the following patents; method and devices for transdermal delivery of lithium (US 6,375,990B1); method of assessing antidepressant drug therapy via transport inhibition of monoamine neurotransmitters by ex vivo assay (US 7,148,027B2). RRB is an employee of Blue Cross of Idaho. The remaining authors declare no potential conflicts of interest.

\section{ACKNOWLEDGMENTS}

The ideas in this article were generated during discussions held at the Banbury Center, Cold Spring Harbor Laboratory in September 2015, 'Therapeutic Use of Ketamine for Treating Severe Depression: Risks and Potential'. The authors would like to acknowledge others that have contributed to the discussions related to this manuscript, including Glen Z. Brooks, Dennis Charney, Maurizio Fava, Howard Goldman, John H. Krystal, Anil K. Malhotra, Steven P. Levine, Alan Schatzberg, Paul Summergrad, and Mason Turner.

\section{REFERENCES}

Advisory Council on the Misuse of Drugs (2013). Ketamine: a review of use and harm. Available at: https://www.gov.uk/government/ uploads/system/uploads/attachment_data/file/264677/ACMD_keta mine_report_dec13.pdf. Accessed 10 December 2013.

Duman RS, Aghajanian GK, Sanacora G, Krystal JH (2016). Synaptic plasticity and depression: new insights from stress and rapid-acting antidepressants. Nat Med 22: 238-249.

Murrough JW, Perez AM, Pillemer S, Stern J, Parides MK, aan het Rot $M$ et al (2013). Rapid and longer-term antidepressant effects of repeated ketamine infusions in treatment-resistant major depression. Biol Psychiatry 74: 250-256.

Newport DJ, Carpenter LL, McDonald WM, Potash JB, Tohen M, Nemeroff CB et al (2015). Ketamine and other NMDA antagonists: early clinical trials and possible mechanisms in depression. Am J Psychiatry 172: 950-966.

Sanacora G, Schatzberg AF (2015). Ketamine: promising path or false prophecy in the development of novel therapeutics for mood disorders? Neuropsychopharmacology 40: 259-267.

Singh JB, Fedgchin M, Daly EJ, De Boer P, Cooper K, Lim P et al (2016). A double-blind, randomized, placebo-controlled, dose-frequency study of intravenous ketamine in patients with treatment-resistant depression. Am J Psychiatry 173: 816-826.

Zanos P, Moaddel R, Morris PJ, Georgiou P, Fischell J, Elmer GI et al (2016). NMDAR inhibition-independent antidepressant actions of ketamine metabolites. Nature 533: 481-486. 